\title{
M-theory and type IIB from a duality manifest action
}

\author{
Chris D.A. Blair, ${ }^{a}$ Emanuel Malek $^{a}$ and Jeong-Hyuck Park ${ }^{a, b}$ \\ ${ }^{a}$ DAMTP, Centre for Mathematical Sciences, University of Cambridge, \\ Wilberforce Road, Cambridge CB3 0WA, United Kingdom \\ ${ }^{b}$ Department of Physics, Sogang University, \\ Mapo-gu, Seoul 121-742, Korea \\ E-mail: C.D.A.Blair@damtp.cam.ac.uk, E.Malek@damtp.cam.ac.uk, \\ Park@sogang.ac.kr
}

AbStract: We revisit the SL(5) U-duality manifest action constructed by Berman and Perry in an extended spacetime. Upon choosing a four-dimensional solution to the section condition constraint, the theory reduces to a four-dimensional truncation of elevendimensional supergravity. In this paper, we show that the theory contains more than this M-theory reduction. The section condition also admits an SL(5) inequivalent threedimensional solution, upon which the action directly reduces to a three-dimensional truncation of type IIB supergravity. We also discuss the reduction to IIB* supergravity.

KeYwords: String Duality, F-Theory, M-Theory

ARXIV EPRINT: 1311.5109

\footnotetext{
${ }^{1}$ On sabbatical leave.
} 


\section{Contents}

1 Introduction 1

2 The duality manifest SL(5) theory 3

2.1 The action, its symmetries and the section condition 4

2.2 M-theory section 5

3 Type IIB supergravity from the duality manifest action 5

3.1 IIB section 5

$\begin{array}{ll}3.2 & \text { Reduction to IIB supergravity }\end{array}$

4 Lorentzian M/IIB and $\mathrm{M}^{*} / \mathrm{IIB}^{*}$ theories $\quad 8$

$\begin{array}{lll}5 & \text { Discussion } & 9\end{array}$

$\begin{array}{ll}\text { A Non-linear realisations and the generalised metric } & 10\end{array}$

B Conventions and useful formulae $\quad 12$

$\begin{array}{ll}\text { B.1 Conventions for spacetime curvature } & 12\end{array}$

B.2 Alternative form of the duality manifest action 12

\section{Introduction}

The presence of string theory dualities leads to the idea of M-theory as a unification of all superstring theories $[1,2]$. These dualities map between different theories, and allow one to identify individual string theories as limits of M-theory.

For instance, type IIA string theory can be viewed as M-theory compactified on a circle. In order to further make an identification with type IIB we have to compactify a second direction and use T-duality. In this paper, working within the context of the low energy effective theories, we will show how a recently developed formulation that makes dualities manifest allows one to directly identify M-theory/IIA and IIB within a single framework.

In doing so we draw inspiration from several different approaches to understanding string theories and their low energy actions through dualities. The common theme of these approaches - reminiscent of the introduction of M-theory as a higher-dimensional theory allowing one to understand features of the original superstring - is to view dualities as manifest symmetries of some larger, often geometric, structure, which reduces to or contains the initial theory of interest.

F-theory [3] provides a realisation of IIB as a reduction from a higher-dimensional theory. The extra dimensions geometrise the SL(2) S-duality of IIB supergravity so that one has a twelve-dimensional theory. There were hints that this geometrisation could be extended to the U-dualities of type IIB [4]. 
More recently, the introduction of generalised geometry by Hitchin and Gualtieri $[5,6]$ has provided a framework in which one makes dualities manifest by extending the tangent bundle $T M$. For T-dualities [7-9] one studies the extended tangent bundle $T M \oplus T^{*} M$, allowing one to combine diffeomorphisms and B-field gauge transformations. The extra factor $T^{*} M$ is needed to take into account the effects of string windings. A natural object appearing on the extended tangent bundle is the generalised metric, originally introduced in [10], which combines the metric and $B$-field into a T-duality covariant object.

By further adding appropriate bundles for D-brane wrappings one arrives at generalised geometries that describe U-duality in type II theories [11-14]. In this approach the IIA and IIB theories have to be treated differently, i.e. they require different choices of generalised geometries. Their extended tangent bundles are $T M \oplus T^{*} M \oplus \Lambda^{\text {even/odd }} T^{*} M \oplus \cdots$, with the even/odd exterior products of the cotangent bundle corresponding to IIA/IIB. One can also extend this treatment to M-theory [14-17], where one now has wrappings of the M2 and M5 branes. The corresponding extended tangent bundle is $T M \oplus \Lambda^{2} T^{*} M \oplus \Lambda^{5} T^{*} M \oplus \cdots$. One can see that in this approach type IIA arises from the usual reduction of M-theory, whereas a different extended tangent bundle is used for IIB.

In order to make the dualities manifest in the action, one can introduce extra coordinates, as pioneered by Duff, Tseytlin and Siegel in the early 90s [10, 18-22]. The recent revival of these ideas has led to a T-duality manifest rewriting of ten-dimensional supergravity known as double field theory [23-39]. An analogous programme exists for M-theory and eleven-dimensional supergravity [40-48], and makes the U-dualities manifest symmetries of the theory. In the extended theories, the fields do not however depend on all of the coordinates. All physical fields and gauge parameters have to satisfy the "section condition" which reduces their dependence and allows one to return to the familiar unextended theory. In fact, imposing the section condition can be shown to be equivalent to "gauging" the extended coordinate space $[49,50]$.

One might argue that due to the section condition, these extended theories are locally equivalent to the description of generalised geometry $[7,15,16]$ where no extra coordinates are introduced. In this paper we will show that the U-duality extended theory encompasses more than just the generalised geometry for M-theory from which it was constructed. It contains a new solution to the section condition which gives rise directly to the IIB theory. We will show how this works explicitly for the SL(5) duality group. We note that a similar solution to the section condition exists for the case of $E_{6}$ [51].

The reduction to IIB is achieved when the generalised metric is taken to be the fundamental object rather than the supergravity fields themselves. These only arise upon choosing a specific parameterisation. If one had viewed the supergravity fields as fundamental, one could not have obtained a reduction to IIB. This approach allows both type II theories to appear within the same duality manifest theory. ${ }^{1}$

\footnotetext{
${ }^{1}$ For T-duality, the IIA and IIB theories can be treated equally in the generalised geometry approach [1114] and in double field theory [22, 36, 38]. Furthermore, they can be unified using $\mathcal{N}=2, D=10$ supersymmetric double field theory [34] where there is no distinction between IIA and IIB thanks to the doubling of the local Lorentz groups, $\operatorname{Spin}(1,9) \rightarrow \operatorname{Spin}(1,9)_{L} \times \operatorname{Spin}(1,9)_{R}$. This supersymmetric theory is unique while its solutions can be classified into IIA and IIB, which are therefore unified.
} 
We note that IIB appears here in a similar spirit to how it arises in F-theory. While in F-theory one introduces an extra two-dimensional space to geometrise the IIB S-duality, we are dealing with a larger U-duality group and so will use a larger extended space. As in F-theory there will be no dependence on the extra coordinates, but they are still crucial for realising the duality manifestly. Our extra coordinates will be in one-to-one correspondence with the wrapping modes of the branes of the IIB theory.

That it should be possible to treat M-theory and IIB in this similar way has been previously suggested by the $E_{11}$ framework of West et al. [52-64] in which it is argued that both eleven-dimensional supergravity and the ten-dimensional IIA and IIB supergravities can be understood as non-linear realisations of $E_{11}$. In particular by identifying the generators of the algebra corresponding to IIA and IIB fields, a relation between the two supergravities appears directly at the ten-dimensional level $[57,59]$. The approach we will take in this paper allows us to realise this idea concretely at the level of the action.

For the sake of simplicity, we will work solely with the SL(5) duality manifest theory [40-42, 44-46], originally developed for a four-dimensional truncation of elevendimensional supergravity. ${ }^{2}$ We will show how in this theory a three-dimensional truncation of type IIB supergravity arises naturally, alongside a four-dimensional truncation of eleven-dimensional supergravity (which can be further reduced to a truncation of type IIA supergravity).

We begin by briefly reviewing the key ingredients of the duality manifest field theory of $\mathrm{SL}(5)$ in section 2. In section 3 we then show that the section condition has a new type of solution which cannot be transformed by means of an SL(5) duality into the M-theory solution previously studied in the literature. Our new solution thus defines a distinct corner of the duality manifest theory which, as we will show, corresponds to the type IIB theory. In section 4 we discuss what happens when one includes timelike directions. We find not

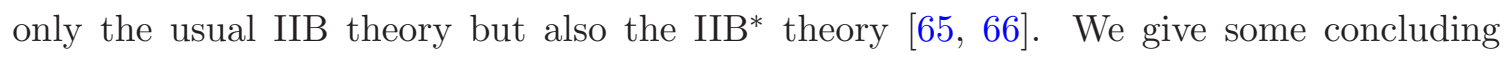
comments in section 5 .

\section{The duality manifest SL(5) theory}

The SL(5) theory we use was originally formulated in [40] by embedding a four-dimensional truncation of eleven-dimensional supergravity into an extended space. We will however present a "top-down" approach in order to emphasise that we view the generalised metric as fundamental and not the supergravity fields which only arise through a specific parameterisation. This is crucial in order to obtain the IIB theory in section 3. Note that for now we take our spaces to be Euclidean for convenience, and will deal with timelike directions in section 4.

\footnotetext{
${ }^{2}$ Historically, SL(5) is viewed as the duality group that controls the scalar sector arising from a sevendimensional truncation of eleven-dimensional supergravity. One assumes there is no dependence on the four dimensions in which the $\mathrm{SL}(5)$ can be seen to act. The convention in the duality manifest theories is different: here the truncation is taken so that no fields depend on the seven directions and because of the introduction of extended coordinates, the SL(5) symmetry becomes manifest.
} 


\subsection{The action, its symmetries and the section condition}

To construct an SL(5) duality manifest action, one uses a ten-dimensional coordinate space such that the coordinates, $x^{a b}=-x^{b a}$, lie in the antisymmetric representation of SL(5). Here $a, b=1, \ldots, 5$ are fundamental SL(5) indices. A $10 \times 10$ generalised metric $[15,18,40]$ acts on this coordinate space and parameterises the coset $\mathbb{R}^{+} \times \mathrm{SL}(5) / \mathrm{SO}(5)$ in which the bosonic supergravity fields we are interested in are known to live. By restricting to SL(5) dualities we have implicitly performed a truncation from some larger duality group, and this truncation gives rise to an extra scalar $[14,17,46,47]$ corresponding to the $\mathbb{R}^{+}$factor. ${ }^{3}$ We discuss this in appendix A.

As a consequence of the coset condition the generalised metric of the SL(5) theory can be decomposed in terms of a "little metric" $m_{a b}$ [41]. This is a symmetric $5 \times 5$ matrix which is a rank two tensor under SL(5) U-dualities.

We can use this little metric to write the duality manifest action controlling the dynamics of this theory $[40,46]$

$$
\begin{aligned}
S= & \int_{\Sigma}|m|^{-1}\left(-\frac{1}{8} m^{a b} m^{a^{\prime} b^{\prime}} \partial_{a a^{\prime}} m^{c d} \partial_{b b^{\prime}} m_{c d}+\frac{1}{2} m^{a b} m^{a^{\prime} b^{\prime}} \partial_{a a^{\prime}} m^{c d} \partial_{c b^{\prime}} m_{b d}\right. \\
& +\frac{1}{2} \partial_{a a^{\prime}} m^{a b} \partial_{b b^{\prime}} m^{a^{\prime} b^{\prime}}+\frac{3}{8} m^{a b} m^{a^{\prime} b^{\prime}} \partial_{a a^{\prime}} \ln |m| \partial_{b b^{\prime}} \ln |m|-2 m^{a^{\prime} b^{\prime}} \partial_{a a^{\prime}} m^{a b} \partial_{b b^{\prime}} \ln |m| \\
& \left.+m^{a^{\prime} b^{\prime}} \partial_{a a^{\prime}} \partial_{b b^{\prime}} m^{a b}-m^{a b} m^{a^{\prime} b^{\prime}} \partial_{a a^{\prime}} \partial_{b b^{\prime}} \ln |m|\right) .
\end{aligned}
$$

Here $\Sigma$ is some lower-dimensional section of the full ten-dimensional theory, and $m \equiv$ $\operatorname{det} m_{a b}$ is used to define the SL(5) singlet integral measure, $|m|^{-1}[46]$.

The infinitesimal symmetries of this action are generalised diffeomorphisms, given by the generalised Lie derivative $[41,67]$

$$
\mathcal{L}_{\xi} m_{a b}=\frac{1}{2} \xi^{c d} \partial_{c d} m_{a b}-\frac{1}{2} m_{a b} \partial_{c d} \xi^{c d}+m_{c b} \partial_{a d} \xi^{c d}+m_{a c} \partial_{b d} \xi^{c d}
$$

Unlike in conventional Riemannian geometry, the algebra of this generalised Lie derivative does not automatically close. To obtain closure and a consistent theory, one must impose a constraint known as the section condition [41]

$$
\partial_{[a b} \partial_{c d]} \Phi(x)=0, \quad \partial_{[a b} \Phi(x) \partial_{c d]} \Phi^{\prime}(x)=0,
$$

for any fields $\Phi(x), \Phi^{\prime}(x)$ in the theory.

The action of (2.1) is the most general two-derivative action involving $m_{a b}$ that is invariant under these local symmetries up to the section condition. It is related to the action in [40] by an integration by parts. It can also be given a "U-geometric" interpretation [46] as a scalar curvature of generalised covariant derivatives, (B.4) (see also [47, 48] for other U-duality groups).

\footnotetext{
${ }^{3}$ We view this $\mathbb{R}^{+}$factor as arising from a truncation of a larger duality group - alternatively, it can be thought of as a scaling symmetry as in [14].
} 


\subsection{M-theory section}

The solution of the section condition (2.3) which gives rise to a four-dimensional truncation of eleven-dimensional supergravity is [40]

$$
\partial_{\alpha \beta}=0, \quad \partial_{\alpha 5} \neq 0,
$$

where $\alpha, \beta=1, \ldots, 4$. To see this, we use a convenient parameterisation of $m_{a b}$ encoding the metric, three-form and an extra scalar as $[41,46]$

$$
m_{a b}=\left(\begin{array}{cc}
\frac{g_{\alpha \beta}}{\sqrt{|g|}} & v_{\alpha} \\
v_{\beta} & \sqrt{|g|}\left(e^{\phi}+v^{\alpha} v_{\alpha}\right)
\end{array}\right) .
$$

Here we have dualised the three-form field by

$$
v^{\alpha}=\frac{1}{3 !} \epsilon^{\alpha \beta \gamma \delta} C_{\beta \gamma \delta},
$$

where $\epsilon^{\alpha \beta \gamma \delta}$ is the four-dimensional Levi-Civita tensor, $\epsilon^{1234}=|g|^{-1 / 2}$. The scalar degree of freedom, $\phi$, is needed for the truncation from eleven dimensions to be duality covariant. Without this scalar the action of SL(5) on $m_{a b}$ does not close [46]. One can understand $\phi$ as coming from the truncation of the other seven directions by constructing the generalised metric as a non-linear realisation of $E_{11}$ [42] as we will discuss in appendix A. Let us stress that $m_{a b}$ is the fundamental object, not the supergravity fields, $g_{\alpha \beta}, C_{\alpha \beta \gamma}, \phi$, themselves. They appear here upon choosing a parameterisation for the M-theory section. As we show in section 3 , the reduction to IIB uses a different parameterisation.

Using the solution of the section condition (2.4) and the parameterisation (2.5) the action reduces to $[41,46]$

$$
S_{4 d}=-\int d^{4} x e^{-2 \phi} \sqrt{|g|}\left(R-\frac{1}{48} e^{-\phi} F_{\alpha \beta \gamma \delta} F^{\alpha \beta \gamma \delta}+\frac{5}{2} \partial_{\alpha} \phi \partial^{\alpha} \phi\right),
$$

where $F_{\alpha \beta \gamma \delta}$ is the field strength of the three-form potential,

$$
F_{\alpha \beta \gamma \delta}=4 \partial_{[\alpha} C_{\beta \gamma \delta]} .
$$

This action is a truncation of the eleven-dimensional supergravity action with the scalar field related to the warping of the other seven dimensions. One could introduce a further isometry and perform a Kaluza-Klein reduction of the theory to a three-dimensional truncation of IIA supergravity.

\section{Type IIB supergravity from the duality manifest action}

In this section, we will exhibit a new solution to the section condition allowing one to reduce to IIB supergravity. Thus, the duality manifest theory with its extended coordinate space allows one to treat M-theory/IIA and IIB in a unified manner.

\subsection{IIB section}

This is a novel three-dimensional section which up to $\mathrm{SL}(5)$ transformations can be written as

$$
\partial_{\mu i}=0, \quad \partial_{i j}=0,
$$

where $\mu, \nu=1,2,3$ and $i, j=4,5$. Thus the solution only depends on $x^{12}, x^{23}, x^{31}$. 
This section and the M-theory section (2.4) are inequivalent, i.e. they cannot be transformed into each other by SL(5). A way of seeing this is to note that $\partial_{[a b} \Phi \partial_{c][d} \Phi^{\prime} \partial_{e f]} \Phi^{\prime \prime}=0$ for the M-theory section, but $\partial_{[a b} \Phi \partial_{c][d} \Phi^{\prime} \partial_{e f]} \Phi^{\prime \prime} \neq 0$ for the IIB section, where $\Phi, \Phi^{\prime}, \Phi^{\prime \prime}$ are any three fields in the theory. As $\partial_{[a b} \Phi \partial_{c][d} \Phi^{\prime} \partial_{e f]} \Phi^{\prime \prime}$ transforms as an SL(5) tensor, the inequivalence of the two sections follows.

\subsection{Reduction to IIB supergravity}

We now show that the new section (3.1) should indeed be labelled as a "IIB section". Here $\mu, \nu=1,2,3$ are three-dimensional spacetime indices and $i, j=4,5$ are $\mathrm{SL}(2)$ S-duality indices. Our fields depend only on the coordinates $x^{\mu \nu}$. To interpret these more naturally as spacetime coordinates we dualise

$$
\tilde{x}_{\mu} \equiv \frac{1}{2} \eta_{\mu \nu \rho} x^{\nu \rho}, \quad \tilde{\partial}^{\mu} \equiv \frac{1}{2} \eta^{\mu \nu \rho} \partial_{\nu \rho}
$$

where $\eta_{123}=\eta^{123}=1$ is the three-dimensional Levi-Civita tensor density.

We now parameterise the little metric $m_{a b}$ to reflect the split defined by this section:

$$
m_{a b}=\left(\begin{array}{cc}
\sqrt{|\tilde{g}|}\left(\tilde{g}_{\mu \nu}+e^{\tilde{\phi}} \tilde{v}^{k}{ }_{\mu} \tilde{v}_{k \nu}\right) & e^{\tilde{\phi}} \tilde{v}_{j \mu} \\
e^{\tilde{\phi}} \tilde{v}_{i \nu} & \frac{1}{\sqrt{|\tilde{g}|}} e^{\tilde{\phi}} \tilde{\mathcal{M}}_{i j}
\end{array}\right) .
$$

This parameterisation is by no means unique, but it provides a convenient way to make a connection with type IIB supergravity.

All the fields appearing in (3.3) can be understood as "dual fields" in the sense of [68-78]. To be consistent with the lower index of the dual coordinates, $\tilde{x}_{\mu}$, the spacetime metric is $\tilde{g}^{\mu \nu}$ with upper indices. The determinant is defined to be $\tilde{g}=\operatorname{det} \tilde{g}^{\mu \nu}$. The Kalb-Ramond and Ramond-Ramond 2-forms combine into an SL(2) doublet $\tilde{C}^{i \mu \nu}$, which appears in the little metric in dualised form:

$$
\tilde{v}^{i}{ }_{\mu}=\frac{1}{2} \tilde{\epsilon}_{\mu \nu \rho} \tilde{C}^{i \nu \rho}
$$

where $\tilde{\epsilon}_{\mu \nu \rho}$ is the three-dimensional Levi-Civita tensor with $\tilde{\epsilon}_{123}=|\tilde{g}|^{-1 / 2}$.

In addition, we have a symmetric $2 \times 2$ unit determinant matrix $\tilde{\mathcal{M}}_{i j}$ which we use to raise and lower SL(2) indices. This matrix can be built out of the Ramond-Ramond scalar $\tilde{C}^{(0)}$ and the string dilaton $\tilde{\varphi}$. A conventional parameterisation is e.g. [79]

$$
\tilde{\mathcal{M}}_{i j}=\frac{1}{\operatorname{Im} \tau}\left(\begin{array}{cc}
|\tau|^{2} & \operatorname{Re} \tau \\
\operatorname{Re} \tau & 1
\end{array}\right), \quad \tau=\tilde{C}^{(0)}+i e^{-\tilde{\varphi}} .
$$

Finally, as in the M-theory reduction (2.5), we have an extra scalar $\tilde{\phi}$, resulting from the truncation of the other seven dimensions, as discussed in appendix A. Once again, this extra scalar is necessary for the action of SL(5) to close [46].

The generalised Lie derivative (2.2) of the little metric now gives us the expected gauge symmetries of the IIB fields. We have

$$
\mathcal{L}_{\xi} m_{a b}=\frac{1}{2} \xi^{c d} \partial_{c d} m_{a b}-\frac{1}{2} m_{a b} \partial_{c d} \xi^{c d}+m_{c b} \partial_{a d} \xi^{c d}+m_{a c} \partial_{b d} \xi^{c d}
$$


where the parameter of generalised diffeomorphisms can be decomposed into IIB language as

$$
\xi^{a b} \rightarrow\left(\xi^{\mu \nu}, \xi^{i \mu}, \xi^{i j}\right)
$$

For the IIB parameterisation we may dualise $\xi^{\mu \nu}$ to $\tilde{\xi}_{\mu}$ and take this to parameterise three-dimensional diffeomorphisms. We also interpret $\xi^{i \mu} \equiv \lambda^{i \mu}$ as corresponding to gauge transformations of the two-forms, while the final component $\xi^{i j}$ actually drops out of the generalised Lie derivative. With this understanding, the generalised Lie derivative (3.6) decomposes to give

$$
\begin{aligned}
\delta \tilde{\phi} & =\tilde{\xi}_{\lambda} \tilde{\partial}^{\lambda} \tilde{\phi}=\mathcal{L}_{\tilde{\xi}} \tilde{\phi}^{\phi} \\
\delta \tilde{\mathcal{M}}_{i j} & =\tilde{\xi}_{\lambda} \tilde{\partial}^{\lambda} \tilde{\mathcal{M}}_{i j}=\mathcal{L}_{\tilde{\xi}} \tilde{\mathcal{M}}_{i j}, \\
\delta \tilde{C}^{i \mu \nu} & =\tilde{\xi}_{\lambda} \tilde{\partial}^{\lambda} \tilde{C}^{i \mu \nu}+\tilde{\partial}^{\mu} \tilde{\xi}_{\lambda} \tilde{C}^{i \lambda \nu}+\tilde{\partial}^{\nu} \tilde{\xi}_{\lambda} \tilde{C}^{i \mu \lambda}+\tilde{\partial}^{\mu} \tilde{\lambda}^{i \nu}-\tilde{\partial}^{\nu} \tilde{\lambda}^{i \mu}=\mathcal{L}_{\tilde{\xi}} \tilde{C}^{i \mu \nu}+2 \tilde{\partial}^{[\mu} \lambda^{|i| \nu]}, \\
\delta \tilde{g}^{\mu \nu} & =\tilde{\xi}_{\lambda} \tilde{\partial}^{\lambda} \tilde{g}^{\mu \nu}+\tilde{\partial}^{\mu} \tilde{\xi}_{\lambda} \tilde{g}^{\lambda \nu}+\tilde{\partial}^{\nu} \tilde{\xi}_{\lambda} \tilde{g}^{\mu \lambda}=\mathcal{L}_{\tilde{\xi}} \tilde{g}^{\mu \nu},
\end{aligned}
$$

where

$$
\mathcal{L}_{\tilde{\xi}} V^{\mu} \equiv \tilde{\xi}_{\nu} \tilde{\partial}^{\nu} V^{\mu}+V^{\nu} \tilde{\partial}^{\mu} \tilde{\xi}_{\nu}
$$

is the Lie derivative for dual diffeomorphisms [72, 75-77].

Having understood the field content and symmetries corresponding to this parameterisation, we are now in a position to work out what our action is. Using the section (3.1) and the parameterisation (3.3) the duality manifest action (2.1) reduces to ${ }^{4}$

$$
S_{3 d}=\int d^{3} \tilde{x} \sqrt{|\tilde{g}|} e^{-2 \tilde{\phi}}\left(-\tilde{R}-\frac{1}{4} \tilde{\partial}^{\mu} \tilde{\mathcal{M}}_{i j} \tilde{\partial}_{\mu} \tilde{\mathcal{M}}^{i j}+\frac{1}{12} e^{\tilde{\phi}} \tilde{H}^{i \mu \nu \rho} \tilde{H}_{i \mu \nu \rho}-\frac{9}{2} \tilde{\partial}^{\mu} \tilde{\phi} \tilde{\partial}_{\mu} \tilde{\phi}\right),
$$

up to total derivatives. This is the expected truncated IIB action, written in terms of the dual fields and involving the extra scalar $\tilde{\phi}$ which is a relic of the truncation. The field strengths appearing here are

$$
\tilde{H}^{i \mu \nu \rho}=3 \tilde{\partial}^{[\mu} \tilde{C}^{|i| \nu \rho]},
$$

which are clearly invariant under the gauge symmetry of $\tilde{C}^{i \mu \nu}$ from (3.8). The unit determinant matrix $\tilde{\mathcal{M}}_{i j}$ contains the dilaton $\tilde{\varphi}$ and $\mathrm{RR}$ zero-form $\tilde{C}^{(0)}$, as for instance given in (3.5). All the $\mathrm{SL}(2)$ indices are contracted with $\tilde{\mathcal{M}}_{i j}$, making the $\mathrm{SL}(2)$ S-duality symmetry of the IIB theory manifest.

Finally, note that in the dual notation, the Ricci scalar $\tilde{R}$ is defined with respect to $\tilde{g}^{\mu \nu}$, i.e. in terms of the Levi-Civita connection $\tilde{\Gamma}_{\rho}^{\mu \nu}=\frac{1}{2} \tilde{g}_{\rho \lambda}\left(\tilde{\partial}^{\mu} \tilde{g}^{\nu \lambda}+\tilde{\partial}^{\nu} \tilde{g}^{\mu \lambda}-\tilde{\partial}^{\lambda} \tilde{g}^{\mu \nu}\right)$ and the Riemann tensor $\tilde{R}_{\mu}{ }^{\nu \rho \sigma}=2 \tilde{\partial}^{[\rho} \tilde{\Gamma}^{\sigma] \nu}{ }_{\mu}+2 \tilde{\Gamma}^{[\rho \mid \lambda}{ }_{\mu} \tilde{\Gamma}^{\mid \sigma] \nu}{ }_{\lambda}$ by $\tilde{R}=\tilde{g}_{\mu \nu} \tilde{R}_{\rho}{ }^{\mu \rho \nu}$. These have been further studied in [70-73, 75-77].

We can also write the action in Einstein frame by rescaling $\tilde{g}_{E}^{\mu \nu}=e^{-4 \tilde{\phi}} \tilde{g}^{\mu \nu}$ to obtain the action

$$
S_{3 d}=\int d^{3} \tilde{x} \sqrt{\left|\tilde{g}_{E}\right|}\left(-\tilde{R}\left(\tilde{g}_{E}\right)-\frac{1}{4} \tilde{\partial}^{\mu} \tilde{\mathcal{M}}_{i j} \tilde{\partial}_{\mu} \tilde{\mathcal{M}}^{i j}+\frac{1}{12} e^{-7 \tilde{\phi}} \tilde{H}^{i \mu \nu \rho} \tilde{H}_{i \mu \nu \rho}+\frac{7}{2} \tilde{\partial}^{\mu} \tilde{\phi} \tilde{\partial}_{\mu} \tilde{\phi}\right)
$$

where all spacetime contractions are now with $\tilde{g}_{E}^{\mu \nu}$.

\footnotetext{
${ }^{4}$ We found the symbolic computing system Cadabra [80, 81] helpful in calculating this reduction, as well as those appearing later in the paper.
} 


\section{Lorentzian $\mathrm{M} / \mathrm{IIB}$ and $\mathrm{M}^{*} / \mathrm{IIB}^{*}$ theories}

We now consider the SL(5) theory relevant for Lorentzian spacetimes. The generalised metric then parameterises the coset space $\mathbb{R}^{+} \times \mathrm{SL}(5) / \mathrm{SO}(3,2)$ [82]. The overall signature of $m_{a b}$ is $(-,+,+,+,-)$ but there is a choice of how to distribute this. Each choice defines different versions of the theory. These correspond not only to the familiar eleven- and tendimensional supergravities, but also to variants of these theories with unusual signatures and kinetic terms coming with the wrong sign, which were first introduced in [65, 66], and have been studied in the context of $E_{11}$ and its decompositions in [83-86].

Firstly, for the M-theory section, the possible parameterisations we should now use are $[46]$

$$
m_{a b}=\left(\begin{array}{cc}
\frac{g_{\alpha \beta}}{\sqrt{|g|}} & v_{\alpha} \\
v_{\beta} & \sqrt{|g|}\left( \pm e^{\phi}+v^{\alpha} v_{\alpha}\right)
\end{array}\right),
$$

where the choice of sign in $\pm e^{\phi}$ depends on the signature of the metric $g_{\alpha \beta}$. There are two possibilities:

Lorentzian M-theory: the signature of $g_{\alpha \beta}$ is $(-,+,+,+)$ and we have to use $-e^{\phi}$ as the scalar term in (4.1). The action in Einstein frame is

$$
S_{4 d}=\int d^{4} x \sqrt{\left|g_{E}\right|}\left(R\left(g_{E}\right)-\frac{1}{48} e^{-7 \phi} F_{\alpha \beta \gamma \delta} F^{\alpha \beta \gamma \delta}-\frac{7}{2} \partial_{\alpha} \phi \partial^{\alpha} \phi\right),
$$

where the Einstein frame metric is related to the one appearing in the little metric (4.1) by $\left(g_{E}\right)_{\alpha \beta}=e^{-2 \phi} g_{\alpha \beta}$, and we are ignoring total derivatives. This is of course the known truncation of the standard eleven-dimensional supergravity [40, 46].

M*-theory: the signature of $g_{\alpha \beta}$ is $(-,-,+,+)$ and we have $+e^{\phi}$. The action in Einstein frame is

$$
S_{4 d}=\int d^{4} x \sqrt{\left|g_{E}\right|}\left(-R\left(g_{E}\right)+\frac{1}{48} e^{-7 \phi} F_{\alpha \beta \gamma \delta} F^{\alpha \beta \gamma \delta}+\frac{7}{2} \partial_{\alpha} \phi \partial^{\alpha} \phi\right) .
$$

The $\mathrm{M}^{*}$-theory has previously been studied in terms of timelike dualities in M-theory [66].

For the three-dimensional IIB section we may again use the parameterisation (3.3), except now there are three possible ways of distributing the signature choices between the spacetime metric $\tilde{g}^{\mu \nu}$ and the $\operatorname{SL}(2)$ scalar matrix $\tilde{\mathcal{M}}_{i j}$. These are:

Lorentzian IIB theory: here the signature of $\tilde{g}^{\mu \nu}$ is $(+,-,-)$ while $\tilde{\mathcal{M}}_{i j}$ has signature $(+,+)$. We obtain the action of the IIB theory in Einstein frame:

$S_{3 d}=\int d^{3} \tilde{x} \sqrt{\left|\tilde{g}_{E}\right|}\left(-\tilde{R}\left(\tilde{g}_{E}\right)-\frac{1}{4} \tilde{\partial}_{\mu} \tilde{\mathcal{M}}_{i j} \tilde{\partial}^{\mu} \tilde{\mathcal{M}}^{i j}+\frac{1}{12} e^{-7 \tilde{\phi}} \tilde{H}_{\mu \nu \rho}^{i} \tilde{H}^{j \mu \nu \rho} \tilde{\mathcal{M}}_{i j}+\frac{7}{2} \tilde{\partial}_{\mu} \tilde{\phi} \partial^{\mu} \tilde{\phi}\right)$.

Note that $-\tilde{R}$ is the expected Ricci scalar term for the mostly negative metric (see appendix B for our conventions), and so all the terms in this action come with the expected right signs to give us a truncation of IIB supergravity theory. 
IIB* theory: the signature of $\tilde{g}^{\mu \nu}$ is $(-,+,+)$ while $\tilde{\mathcal{M}}_{i j}$ has signature $(-,+)$ (and so parameterises the coset $\mathrm{SL}(2) / \mathrm{SO}(1,1)$ rather than $\mathrm{SL}(2) / \mathrm{SO}(2))$. The Einstein frame action is

$$
S_{3 d}=\int d^{3} \tilde{x} \sqrt{\mid \tilde{g}_{E}}\left(\tilde{R}\left(\tilde{g}_{E}\right)+\frac{1}{4} \tilde{\partial}_{\mu} \tilde{\mathcal{M}}_{i j} \tilde{\partial}^{\mu} \tilde{\mathcal{M}}^{i j}+\frac{1}{12} e^{-7 \tilde{\phi}} \tilde{H}^{i}{ }_{\mu \nu \rho} \tilde{H}^{j \mu \nu \rho} \tilde{\mathcal{M}}_{i j}-\frac{7}{2} \tilde{\partial}_{\mu} \tilde{\phi} \partial^{\mu} \tilde{\phi}\right) .
$$

We note that one of the scalars of $\tilde{\mathcal{M}}_{i j}$ and one of the two-forms $\tilde{C}^{i \mu \nu}$ have kinetic terms with the wrong signs. This corresponds to the type IIB* theory $[65,66]$ which has previously been argued to arise by acting with a timelike duality on IIA.

Euclidean IIB theory: the signature of $\tilde{g}^{\mu \nu}$ is $(+,+,+)$ while $\tilde{\mathcal{M}}_{i j}$ has signature $(-,-)$. The action in Einstein frame becomes

$$
S_{3 d}=\int d^{3} \tilde{x} \sqrt{\tilde{g}_{E}}\left(-\tilde{R}\left(\tilde{g}_{E}\right)-\frac{1}{4} \tilde{\partial}_{\mu} \tilde{\mathcal{M}}_{i j} \tilde{\partial}^{\mu} \tilde{\mathcal{M}}^{i j}+\frac{1}{12} e^{-7 \tilde{\phi}} \tilde{H}^{i}{ }_{\mu \nu \rho} \tilde{H}^{j \mu \nu \rho} \tilde{\mathcal{M}}_{i j}+\frac{7}{2} \tilde{\partial}_{\mu} \tilde{\phi} \partial^{\mu} \tilde{\phi}\right) .
$$

Note that this Euclidean IIB theory is different from (3.12) because $\tilde{\mathcal{M}}_{i j}$ has the opposite signature to the one considered there. This exhausts all the different possibilities.

The $\mathrm{M}^{*}$-theory and $\mathrm{IIB}^{*}$ theory were originally considered in $[65,66]$. There it was argued that one obtains IIB* by starting with IIA and applying a T-duality in the timelike direction. Similarly, there is a IIA* related by a timelike T-duality to the usual IIB theory. In both these theories one finds RR fields whose kinetic terms come with a wrong sign. One can further view $\mathrm{M}^{*}$-theory as being an eleven-dimensional theory with two timelike directions - compactifying on one of these timelike directions produces the IIA* theory.

In the duality covariant picture, we see these theories arising on the same footing, corresponding to different possible choices for the parameterisation of the generalised metric. However, they cannot be related to each other by dualities [69].

\section{Discussion}

In this paper we showed that the SL(5) duality manifest theory contains both M-theory and IIB as different solutions of its section condition. These solutions cannot be related by SL(5) transformations, and therefore represent inequivalent sectors of the theory. In order to find both M-theory and IIB one has to view the generalised metric as fundamental and not the supergravity fields themselves. As a result the duality manifest theory treats the type II theories on the same footing.

By considering the different possible choices of spacetime signature allowed by the coset structure $\mathrm{SL}(5) / \mathrm{SO}(3,2)$ we were able to find reductions to variants of eleven-dimensional supergravity and IIB supergravity. These variants include the normal theories as well as the $\mathrm{M}^{*}$-theory, which has two timelike directions, and the IIB* theory, for which the kinetic terms of some fields have the wrong sign. These theories are disconnected subsectors of the full extended theory, and one cannot transform between them by dualities.

In this paper, we considered only the duality group SL(5) and so obtained three- and four-dimensional truncations of the ten- and eleven-dimensional supergravities. The work 
presented here should be extended to the other duality groups $\mathrm{SO}(5,5), E_{6}$ and $E_{7}$ for which all the ingredients needed for a similar analysis are known [42,67]. Indeed, the existence of an inequivalent IIB section for $E_{6}$ has already been mentioned in [51]. Eventually, one may wish to address this question using the full mechanics of $E_{11}$ where independent evidence $[52-57,59-64]$ has shown that the full ten-dimensional IIA and IIB supergravities should be contained on an equal footing, just as in $\mathcal{N}=2 D=10$ supersymmetric double field theory [34] for T-duality.

As the section constraint is equivalent to gauging the extended coordinate space [49, 50], the choice of a section corresponds to a gauge fixing. In the usual Yang-Mills theories, different gauge fixings are BRST equivalent. It would be interesting to further investigate the BRST-cohomological aspect of the two SL(5) inequivalent sections that we considered here.

\section{Acknowledgments}

We wish to thank Malcolm Perry, Alasdair Routh and Yoonji Suh for helpful discussions. We also thank Henning Samtleben for drawing our attention to a related work. JHP deeply acknowledges the hospitality and encouragement from Hugh Osborn at DAMTP, Cambridge during his sabbatical visit. JHP is supported by the National Research Foundation of Korea (NRF) grant funded by the Korea government (MSIP) with the Grant No. 2012R1A2A2A02046739 and No. 2013R1A1A1A05005747. CB is supported by the STFC, the Cambridge Home and EU Scholarship Scheme and St John's College, Cambridge.

\section{A Non-linear realisations and the generalised metric}

By appropriately decomposing the eleven-dimensional U-duality group $E_{11}$ [42], one can construct generalised metrics for different duality groups. In the case we are concerned with in this paper, we decompose $E_{11}$ to $\mathrm{SL}(5) \times \mathrm{GL}(7)$. This can be done in two ways: one corresponding to M-theory and the other to type IIB. In both cases the generalised metric parameterises the coset space $(\mathrm{SL}(5) \times \mathrm{GL}(7)) /(\mathrm{SO}(5) \times \mathrm{SO}(7))$ (modulo assignment of the timelike direction in spacetime) and can then be constructed as a non-linear realisation of the duality group. One finds $[42,68]$

$$
\mathcal{H}_{M N}=\left|\tilde{g}_{7}\right|^{-1 / 2}|\tilde{g}|^{-1 / 2}\left(\begin{array}{cc}
\tilde{M}_{a b, c d} & 0 \\
0 & \left(\tilde{g}_{7}\right)_{A B}
\end{array}\right) .
$$

Here the indices $M, N$ run over the full $7+10$ dimensional extended space, while $A, B$ are indices in the seven dimensions which will be truncated in what follows and $a, b, \ldots$ as before are fundamental SL(5) indices. The $10 \times 10$ matrix $\tilde{M}_{a b, c d}$ carries pairs of antisymmetric such indices and is what is actually normally referred to as the generalised metric. It will have some particular parameterisation in terms of spacetime fields and in particular its determinant is $\operatorname{det} \tilde{M}=|\tilde{g}|^{-2}$, with $|\tilde{g}|$ being the determinant of the metric of the threeor four-dimensional truncation of spacetime which we are going to restrict to. Finally 
$\left(\tilde{g}_{7}\right)_{A B}$ is the metric for the other seven directions. ${ }^{5}$ We are assuming a block diagonal decomposition of the full spacetime metric, and also that all components of form fields are zero when any index corresponds to one of these seven directions.

The full generalised metric $\mathcal{H}_{M N}$ is a weighted SL(5) tensor, and can be used to directly construct a Lagrangian density. However, we want to truncate to the ten-dimensional extended space. This truncation involves using only the upper left block of the generalised metric $\mathcal{H}_{M N}$. We shall from now on refer to this block as the generalised metric, and denote it

$$
\bar{M}_{a b, c d}=\left|\tilde{g}_{7}\right|^{-1 / 2}|\tilde{g}|^{-1 / 2} \tilde{M}_{a b, c d} .
$$

Neither this generalised metric nor the generalised metric $\tilde{M}_{a b, c d}$ is an element of SL(5), as can be seen by considering their determinants. One can define an SL(5) generalised metric $\hat{M}_{a b, c d}$ with $\operatorname{det} \hat{M}=1$ by $\hat{M}_{a b, c d}=|\tilde{g}|^{1 / 5} \tilde{M}_{a b, c d}$. However, it is not possible to construct the dynamics of the theory using $\hat{M}_{a b, c d}$, as discussed in [42].

We are therefore forced to use generalised metrics which are not truly elements of SL(5). However these generalised metrics will transform correctly under duality transformations by virtue of the factor of the determinant of the metric in the truncated directions appearing outside. It is this $\left|\tilde{g}_{7}\right|^{-1 / 2}$ factor which corresponds to including an extra scalar in [46] and also in our treatment.

The precise way in which one includes this scalar is a matter of taste. One could take a double field theory-esque approach, and define a new scalar density $e^{-2 d}$ by

$$
\bar{M}_{a b, c d}=\left|\tilde{g}_{7}\right|^{-1 / 2}|\tilde{g}|^{-1 / 2} \tilde{M}_{a b, c d}=e^{2 d} \hat{M}_{a b, c d},
$$

where one finds that $e^{2 d}=\left|\tilde{g}_{7}\right|^{-1 / 2}|\tilde{g}|^{-7 / 10}$. Alternatively, the $\left|\tilde{g}_{7}\right|$ factors can be absorbed into field redefinitions.

Explicitly, suppose one decomposes the generalised metric $\tilde{M}_{a b, c d}$ in terms of a little metric $\tilde{m}_{a b}$, so that similarly $\bar{M}_{a b, c d}$ can be written in terms of a little metric $\bar{m}_{a b}$ given by

$$
\bar{m}_{a b}=\left|\tilde{g}_{7}\right|^{-1 / 4}|\tilde{g}|^{-1 / 4} \tilde{m}_{a b} .
$$

We know that the determinant of $\tilde{m}_{a b}$ is $|\tilde{g}|^{-1 / 2}$. We define a new generalised metric in which the factor of $\left|\tilde{g}_{7}\right|^{-1 / 4}$ has been absorbed by

$$
\bar{m}_{a b}=|\operatorname{det} m|^{1 / 2} m_{a b} .
$$

One sees that $m_{a b}$ is unweighted with respect to the generalised Lie derivative and is given by $m_{a b}=\left|\tilde{g}_{7}\right|^{-1 / 14} \tilde{m}_{a b}$.

For both the M-theory and IIB parameterisations it is then a simple matter to determine the precise rescalings of the spacetime fields that make up $\tilde{m}$ : for instance, for the M-theory case this involves equating

$$
m_{a b}=\left(\begin{array}{cc}
\frac{g_{\alpha \beta}}{\sqrt{|g|}} & v_{\alpha} \\
v_{\beta} & \sqrt{|g|}\left( \pm e^{\phi}+v^{\alpha} v_{\alpha}\right)
\end{array}\right)=\left|\tilde{g}_{7}\right|^{-1 / 14}\left(\begin{array}{cc}
\frac{\tilde{g}_{\alpha \beta}}{\sqrt{|\tilde{g}|}} & \tilde{v}_{\alpha} \\
\tilde{v}_{\beta} & \sqrt{|\tilde{g}|}\left( \pm 1+\tilde{v}^{\alpha} \tilde{v}_{\alpha}\right)
\end{array}\right) .
$$

\footnotetext{
${ }^{5}$ In the rest of the paper, we used $\tilde{g}$ to refer to the IIB spacetime metric, to stress that it was given in dual form. However in this appendix $\tilde{g}$ refers to the spacetime metric appearing in the $E_{11}$ generalised $\operatorname{metric}(\mathrm{A} .1)$.
} 
Here $g$ is the spacetime metric that is used for the M-theory truncation throughout the paper. Furthermore, we find here that $e^{\phi}=\left|\tilde{g}_{7}\right|^{-3 / 14}$ while for the IIB generalised metric (3.3) we have $e^{\tilde{\phi}}=\left|\tilde{g}_{7}\right|^{-2 / 7}$. The little metric in the form corresponding to $m_{a b}$ with the scalar $e^{\phi}$ present was first used in [46], and it is this which we use throughout the present work.

\section{B Conventions and useful formulae}

\section{B.1 Conventions for spacetime curvature}

Our spacetime Riemann curvature tensor is defined in terms of the Christoffel symbols $\Gamma_{\mu \nu}{ }^{\rho}$ of the Levi-Civita connection

$$
R_{\nu \rho \sigma}^{\mu}=\partial_{\rho} \Gamma_{\sigma \nu}^{\mu}-\partial_{\sigma} \Gamma_{\rho \nu}^{\mu}+\Gamma_{\rho \lambda}^{\mu} \Gamma_{\sigma \nu}^{\lambda}-\Gamma_{\sigma \lambda}^{\mu} \Gamma_{\rho \nu}{ }^{\lambda},
$$

and the Ricci scalar is

$$
R=g^{\mu \nu} R_{\mu \rho \nu}^{\rho}
$$

With this definition the Einstein-Hilbert Lagrangian for a Lorentzian metric of mostly plus signature is then $+R$, while that for a Lorentzian metric of mostly minus signature is $-R$.

For the dual fields used in the type IIB reduction, these definitions are the same except with the positions of all indices flipped, i.e. $\tilde{R}$ is the Ricci scalar defined with respect to $\tilde{g}^{\mu \nu}$, using the Levi-Civita connection $\tilde{\Gamma}_{\rho}^{\mu \nu}=\frac{1}{2} \tilde{g}_{\rho \lambda}\left(\tilde{\partial}^{\mu} \tilde{g}^{\nu \lambda}+\tilde{\partial}^{\nu} \tilde{g}^{\mu \lambda}-\tilde{\partial}^{\lambda} \tilde{g}^{\mu \nu}\right)$ and the Riemann tensor $\tilde{R}_{\mu}{ }^{\nu \rho \sigma}=2 \tilde{\partial}^{\left[\rho \tilde{\Gamma}^{\sigma] \nu}\right.}{ }_{\mu}+2 \tilde{\Gamma}^{[\rho \mid \lambda}{ }_{\mu} \tilde{\Gamma}^{\mid \sigma] \nu}{ }_{\lambda}$. This has been further studied in [70-73, 75-77].

If $g_{\mu \nu}$ and $\bar{g}_{\mu \nu}=\Omega g_{\mu \nu}$ are two $D$-dimensional metrics related by a conformal scaling then

$$
\bar{R}=\Omega^{-1}\left(R-(D-1) \nabla^{2} \ln \Omega-\frac{1}{4}(D-2)(D-1)(\nabla \ln \Omega)^{2}\right) .
$$

\section{B.2 Alternative form of the duality manifest action}

Following [46], the SL(5) U-duality covariant action (2.1) can be equivalently formulated as

$$
S=\int_{\Sigma}|m|^{-1} \mathcal{R}
$$

where $\mathcal{R}$ is the invariant scalar curvature given by the generalized metric and its derivatives, $\mathcal{R}=\partial_{a b}\left(2 A^{a c b}{ }_{c}-A^{a b c}{ }_{c}\right)+\frac{1}{2} A_{a b c d} A^{a b c d}-2 A_{a b c d} A^{a c b d}-\frac{1}{4} A_{a b c}{ }^{c} A^{a b d}{ }_{d}-2 A_{c a b}{ }^{c} A^{a b d}{ }_{d}+2 A_{c a b}{ }^{c} A^{d b a}{ }_{d}$,

while $A_{a b c d}$ is the simplest choice for a metric-compatible, semi-covariant derivative connection,

$$
A_{a b c d}=A_{[a b](c d)}=\frac{1}{2} m_{c d} m^{e f} \partial_{a b} m_{e f}-\frac{1}{2} \partial_{a b} m_{c d} .
$$

Open Access. This article is distributed under the terms of the Creative Commons Attribution License (CC-BY 4.0), which permits any use, distribution and reproduction in any medium, provided the original author(s) and source are credited. 


\section{References}

[1] C. Hull and P. Townsend, Unity of superstring dualities, Nucl. Phys. B 438 (1995) 109 [hep-th/9410167] [INSPIRE].

[2] E. Witten, String theory dynamics in various dimensions, Nucl. Phys. B 443 (1995) 85 [hep-th/9503124] [INSPIRE].

[3] C. Vafa, Evidence for F-theory, Nucl. Phys. B 469 (1996) 403 [hep-th/9602022] [InSPIRE].

[4] A. Kumar and C. Vafa, U manifolds, Phys. Lett. B 396 (1997) 85 [hep-th/9611007] [INSPIRE].

[5] N. Hitchin, Generalized Calabi-Yau manifolds, Quart. J. Math. Oxford Ser. 54 (2003) 281 [math/0209099] [INSPIRE].

[6] M. Gualtieri, Generalized complex geometry, math/0401221 [INSPIRE].

[7] M. Graña, R. Minasian, M. Petrini and D. Waldram, T-duality, Generalized Geometry and Non-Geometric Backgrounds, JHEP 04 (2009) 075 [arXiv:0807.4527] [InSPIRE].

[8] A. Coimbra, C. Strickland-Constable and D. Waldram, Supergravity as Generalised Geometry I: Type II Theories, JHEP 11 (2011) 091 [arXiv:1107.1733] [INSPIRE].

[9] A. Coimbra, C. Strickland-Constable and D. Waldram, Generalised Geometry and type-II Supergravity, Fortsch. Phys. 60 (2012) 982 [arXiv:1202.3170] [InSPIRE].

[10] M. Duff, Duality Rotations in String Theory, Nucl. Phys. B 335 (1990) 610 [InSPIRE].

[11] M. Graña, J. Louis, A. Sim and D. Waldram, $E_{7(7)}$ formulation of $N=2$ backgrounds, JHEP 07 (2009) 104 [arXiv:0904.2333] [INSPIRE].

[12] G. Aldazabal, E. Andres, P.G. Camara and M. Graña, U-dual fluxes and Generalized Geometry, JHEP 11 (2010) 083 [arXiv: 1007.5509] [INSPIRE].

[13] M. Graña and F. Orsi, N=1 vacua in Exceptional Generalized Geometry, JHEP 08 (2011) 109 [arXiv:1105.4855] [INSPIRE].

[14] A. Coimbra, C. Strickland-Constable and D. Waldram, $E_{d(d)} \times \mathbb{R}^{+}$Generalised Geometry, Connections and M-theory, arXiv:1112.3989 [INSPIRE].

[15] C. Hull, Generalised Geometry for M-theory, JHEP 07 (2007) 079 [hep-th/0701203] [INSPIRE].

[16] P.P. Pacheco and D. Waldram, M-theory, exceptional generalised geometry and superpotentials, JHEP 09 (2008) 123 [arXiv:0804.1362] [INSPIRE].

[17] A. Coimbra, C. Strickland-Constable and D. Waldram, Supergravity as Generalised Geometry II: $E_{d(d)} \times \mathbb{R}^{+}$and M-theory, arXiv:1212.1586 [INSPIRE].

[18] M. Duff and J. Lu, Duality Rotations in Membrane Theory, Nucl. Phys. B 347 (1990) 394 [INSPIRE].

[19] A.A. Tseytlin, Duality Symmetric Formulation of String World Sheet Dynamics, Phys. Lett. B 242 (1990) 163 [INSPIRE].

[20] A.A. Tseytlin, Duality symmetric closed string theory and interacting chiral scalars, Nucl. Phys. B 350 (1991) 395 [InSPIRE].

[21] W. Siegel, Two vierbein formalism for string inspired axionic gravity, Phys. Rev. D 47 (1993) 5453 [hep-th/9302036] [INSPIRE]. 
[22] W. Siegel, Superspace duality in low-energy superstrings, Phys. Rev. D 48 (1993) 2826 [hep-th/9305073] [INSPIRE].

[23] C. Hull and B. Zwiebach, Double Field Theory, JHEP 09 (2009) 099 [arXiv:0904.4664] [INSPIRE].

[24] C. Hull and B. Zwiebach, The gauge algebra of double field theory and Courant brackets, JHEP 09 (2009) 090 [arXiv:0908.1792] [INSPIRE].

[25] O. Hohm, C. Hull and B. Zwiebach, Background independent action for double field theory, JHEP 07 (2010) 016 [arXiv: 1003.5027] [INSPIRE].

[26] O. Hohm, C. Hull and B. Zwiebach, Generalized metric formulation of double field theory, JHEP 08 (2010) 008 [arXiv:1006.4823] [INSPIRE].

[27] O. Hohm and S.K. Kwak, Frame-like Geometry of Double Field Theory, J. Phys. A 44 (2011) 085404 [arXiv:1011.4101] [InSPIRE].

[28] I. Jeon, K. Lee and J.-H. Park, Differential geometry with a projection: Application to double field theory, JHEP 04 (2011) 014 [arXiv:1011.1324] [INSPIRE].

[29] I. Jeon, K. Lee and J.-H. Park, Double field formulation of Yang-Mills theory, Phys. Lett. B 701 (2011) 260 [arXiv:1102.0419] [InSPIRE].

[30] I. Jeon, K. Lee and J.-H. Park, Stringy differential geometry, beyond Riemann, Phys. Rev. D 84 (2011) 044022 [arXiv: 1105.6294] [INSPIRE].

[31] I. Jeon, K. Lee and J.-H. Park, Incorporation of fermions into double field theory, JHEP 11 (2011) 025 [arXiv:1109.2035] [INSPIRE].

[32] I. Jeon, K. Lee and J.-H. Park, Supersymmetric Double Field Theory: Stringy Reformulation of Supergravity, Phys. Rev. D 85 (2012) 081501 [Erratum ibid. D 86 (2012) 089903] [arXiv: 1112.0069] [INSPIRE].

[33] I. Jeon, K. Lee and J.-H. Park, Ramond-Ramond Cohomology and O(D,D) T-duality, JHEP 09 (2012) 079 [arXiv:1206.3478] [INSPIRE].

[34] I. Jeon, K. Lee, J.-H. Park and Y. Suh, Stringy Unification of Type IIA and IIB Supergravities under $N=2 D=10$ Supersymmetric Double Field Theory, Phys. Lett. B 723 (2013) 245 [arXiv: 1210.5078] [INSPIRE].

[35] O. Hohm and S.K. Kwak, Double Field Theory Formulation of Heterotic Strings, JHEP 06 (2011) 096 [arXiv:1103.2136] [INSPIRE].

[36] O. Hohm, S.K. Kwak and B. Zwiebach, Unification of Type II Strings and T-duality, Phys. Rev. Lett. 107 (2011) 171603 [arXiv:1106.5452] [INSPIRE].

[37] O. Hohm, S.K. Kwak and B. Zwiebach, Double Field Theory of Type II Strings, JHEP 09 (2011) 013 [arXiv:1107.0008] [InSPIRE].

[38] O. Hohm and S.K. Kwak, N=1 Supersymmetric Double Field Theory, JHEP 03 (2012) 080 [arXiv: 1111.7293] [INSPIRE].

[39] G. Aldazabal, D. Marques and C. Núñez, Double Field Theory: A Pedagogical Review, Class. Quant. Grav. 30 (2013) 163001 [arXiv:1305.1907] [INSPIRE].

[40] D.S. Berman and M.J. Perry, Generalized Geometry and M-theory, JHEP 06 (2011) 074 [arXiv: 1008.1763] [INSPIRE]. 
[41] D.S. Berman, H. Godazgar, M. Godazgar and M.J. Perry, The local symmetries of M-theory and their formulation in generalised geometry, JHEP 01 (2012) 012 [arXiv:1110.3930] [INSPIRE].

[42] D.S. Berman, H. Godazgar, M.J. Perry and P. West, Duality Invariant Actions and Generalised Geometry, JHEP 02 (2012) 108 [arXiv:1111.0459] [INSPIRE].

[43] D.C. Thompson, Duality Invariance: From M-theory to Double Field Theory, JHEP 08 (2011) 125 [arXiv:1106.4036] [INSPIRE].

[44] D.S. Berman and D.C. Thompson, Duality Symmetric String and M-theory, arXiv: 1306.2643 [INSPIRE].

[45] H. Godazgar, M. Godazgar and M.J. Perry, $E_{8}$ duality and dual gravity, JHEP 06 (2013) 044 [arXiv: 1303.2035] [INSPIRE].

[46] J.-H. Park and Y. Suh, U-geometry: SL(5), JHEP 04 (2013) 147 [arXiv:1302.1652] [INSPIRE].

[47] G. Aldazabal, M. Graña, D. Marqués and J. Rosabal, Extended geometry and gauged maximal supergravity, JHEP 06 (2013) 046 [arXiv:1302.5419] [INSPIRE].

[48] M. Cederwall, J. Edlund and A. Karlsson, Exceptional geometry and tensor fields, JHEP 07 (2013) 028 [arXiv:1302.6736] [INSPIRE].

[49] J.-H. Park, Comments on double field theory and diffeomorphisms, JHEP 06 (2013) 098 [arXiv: 1304.5946] [INSPIRE].

[50] K. Lee and J.-H. Park, Covariant action for a string in doubled yet gauged spacetime, Nucl. Phys. B 880 (2014) 134 [arXiv:1307.8377] [INSPIRE].

[51] O. Hohm and H. Samtleben, Exceptional Form of D = 11 Supergravity, arXiv:1308.1673 [INSPIRE].

[52] P.C. West, $E_{11}$ and M-theory, Class. Quant. Grav. 18 (2001) 4443 [hep-th/0104081] [INSPIRE].

[53] I. Schnakenburg and P.C. West, Kac-Moody symmetries of 2B supergravity, Phys. Lett. B 517 (2001) 421 [hep-th/0107181] [INSPIRE].

[54] P.C. West, Very extended $E_{8}$ and $A_{8}$ at low levels, gravity and supergravity, Class. Quant. Grav. 20 (2003) 2393 [hep-th/0212291] [INSPIRE].

[55] P.C. West, $E_{11}$, SL(32) and central charges, Phys. Lett. B 575 (2003) 333 [hep-th/0307098] [INSPIRE].

[56] A. Kleinschmidt, I. Schnakenburg and P.C. West, Very extended Kac-Moody algebras and their interpretation at low levels, Class. Quant. Grav. 21 (2004) 2493 [hep-th/0309198] [INSPIRE].

[57] P.C. West, The IIA, IIB and eleven-dimensional theories and their common $E_{11}$ origin, Nucl. Phys. B 693 (2004) 76 [hep-th/0402140] [INSPIRE].

[58] A. Kleinschmidt and H. Nicolai, IIB supergravity and $E_{10}$, Phys. Lett. B 606 (2005) 391 [hep-th/0411225] [INSPIRE].

[59] F. Riccioni and P.C. West, The $E_{11}$ origin of all maximal supergravities, JHEP 07 (2007) 063 [arXiv: 0705.0752] [INSPIRE]. 
[60] F. Riccioni and P.C. West, E11-extended spacetime and gauged supergravities, JHEP 02 (2008) 039 [arXiv:0712.1795] [INSPIRE].

[61] F. Riccioni and P. West, Local E $E_{11}$, JHEP 04 (2009) 051 [arXiv: 0902.4678] [InSPIRE].

[62] P.C. West, $E_{11}, \mathrm{SL}(32)$ and central charges, Phys. Lett. B 575 (2003) 333 [hep-th/0307098] [INSPIRE].

[63] P. West, $E_{11}$, generalised space-time and IIA string theory, Phys. Lett. B 696 (2011) 403 [arXiv: 1009.2624] [INSPIRE].

[64] A. Rocen and P. West, E11, generalised space-time and IIA string theory: the RR sector, arXiv:1012.2744 [INSPIRE].

[65] C. Hull, Timelike $T$ duality, de Sitter space, large- $N$ gauge theories and topological field theory, JHEP 07 (1998) 021 [hep-th/9806146] [INSPIRE].

[66] C. Hull, Duality and the signature of space-time, JHEP 11 (1998) 017 [hep-th/9807127] [INSPIRE].

[67] D.S. Berman, M. Cederwall, A. Kleinschmidt and D.C. Thompson, The gauge structure of generalised diffeomorphisms, JHEP 01 (2013) 064 [arXiv:1208.5884] [INSPIRE].

[68] E. Malek, U-duality in three and four dimensions, arXiv:1205.6403 [INSPIRE].

[69] E. Malek, Timelike U-dualities in Generalised Geometry, JHEP 11 (2013) 185 [arXiv: 1301.0543] [INSPIRE].

[70] D. Andriot, M. Larfors, D. Lüst and P. Patalong, A ten-dimensional action for non-geometric fluxes, JHEP 09 (2011) 134 [arXiv:1106.4015] [INSPIRE].

[71] D. Andriot, O. Hohm, M. Larfors, D. Lüst and P. Patalong, A geometric action for non-geometric fluxes, Phys. Rev. Lett. 108 (2012) 261602 [arXiv:1202.3060] [INSPIRE].

[72] D. Andriot, O. Hohm, M. Larfors, D. Lüst and P. Patalong, Non-Geometric Fluxes in Supergravity and Double Field Theory, Fortsch. Phys. 60 (2012) 1150 [arXiv:1204.1979] [INSPIRE].

[73] D. Andriot and A. Betz, $\beta$-supergravity: a ten-dimensional theory with non-geometric fluxes and its geometric framework, JHEP 12 (2013) 083 [arXiv: 1306.4381] [INSPIRE].

[74] R. Blumenhagen, A. Deser, E. Plauschinn and F. Rennecke, Bianchi Identities for Non-Geometric Fluxes - From Quasi-Poisson Structures to Courant Algebroids, Fortsch. Phys. 60 (2012) 1217 [arXiv:1205.1522] [INSPIRE].

[75] R. Blumenhagen, A. Deser, E. Plauschinn and F. Rennecke, A bi-invariant Einstein-Hilbert action for the non-geometric string, Phys. Lett. B 720 (2013) 215 [arXiv:1210.1591] [INSPIRE].

[76] R. Blumenhagen, A. Deser, E. Plauschinn and F. Rennecke, Non-geometric strings, symplectic gravity and differential geometry of Lie algebroids, JHEP 02 (2013) 122 [arXiv: 1211.0030] [INSPIRE].

[77] R. Blumenhagen, A. Deser, E. Plauschinn, F. Rennecke and C. Schmid, The Intriguing Structure of Non-geometric Frames in String Theory, arXiv:1304.2784 [INSPIRE].

[78] R. Blumenhagen, X. Gao, D. Herschmann and P. Shukla, Dimensional Oxidation of Non-geometric Fluxes in Type II Orientifolds, arXiv:1306.2761 [INSPIRE]. 
[79] J. Polchinski, String theory. Vol. 2: Superstring theory and beyond, Cambridge University Press, (1998).

[80] K. Peeters, Introducing Cadabra: A symbolic computer algebra system for field theory problems, hep-th/0701238 [INSPIRE].

[81] K. Peeters, A Field-theory motivated approach to symbolic computer algebra, Comput. Phys. Commun. 176 (2007) 550 [cs/0608005] [inSPIRE].

[82] C. Hull and B. Julia, Duality and moduli spaces for timelike reductions, Nucl. Phys. B 534 (1998) 250 [hep-th/9803239] [INSPIRE].

[83] A. Keurentjes, E $E_{11}$ : Sign of the times, Nucl. Phys. B 697 (2004) 302 [hep-th/0402090] [INSPIRE].

[84] A. Keurentjes, Time-like T duality algebra, JHEP 11 (2004) 034 [hep-th/0404174] [INSPIRE].

[85] S. de Buyl, L. Houart and N. Tabti, Dualities and signatures of $G^{++}$invariant theories, JHEP 06 (2005) 084 [hep-th/0505199] [INSPIRE].

[86] P.P. Cook and P.C. West, $M$-theory solutions in multiple signatures from $E_{11}$, JHEP 12 (2005) 041 [hep-th/0506122] [INSPIRE]. 